OPEN ACCESS

Edited by:

Liren Qian,

Fifth Medical Center of the PLA

General Hospital, China

Reviewed by:

Ashish O. Gupta

University of Minnesota Children's Hospital, United States

Philippe Bourin,

Independent Researcher, Toulouse,

France

Leland Metheny,

University Hospitals of Cleveland,

United States

Giuseppe Alberto Palumbo,

University of Catania, Italy

${ }^{*}$ Correspondence:

Miao Miao

mm85124@sina.com

Depei Wu

wudepei@suda.edu.cn

${ }^{\dagger}$ These authors have contributed equally to this work and share

first authorship

Specialty section: This article was submitted to Hematologic Malignancies,

a section of the journa

Frontiers in Oncology

Received: 09 October 2021 Accepted: 21 December 2021

Published: 24 January 2022

Citation:

Wang Q, Xu N, Wang Y, Zhang X,

Liu L, Zhou H, Wang $H$, Zhang $X$,

Tang X, Fu C, Miao M and Wu D (2022)

Allogeneic Stem Cell Transplantation

Combined With Transfusion of

Mesenchymal Stem Cells in Primary

Myelofibrosis: A Multicenter

Retrospective Study.

Front. Oncol. 11:792142.

doi: 10.3389/fonc.2021.792142

\section{Allogeneic Stem Cell Transplantation Combined With Transfusion of Mesenchymal Stem Cells in Primary Myelofibrosis: A Multicenter Retrospective Study}

\author{
Qingyuan Wang ${ }^{1,2,3 \dagger}$, Na Xu ${ }^{4 \dagger}$, Yu Wang ${ }^{5,6,7}$, Xi Zhang ${ }^{8}$, Limin Liu ${ }^{1,2,3}$, Huifen Zhou ${ }^{1,2,3}$, \\ Hong Wang ${ }^{1,2,3}$, Xiang Zhang ${ }^{1,2,3}$, Xiaowen Tang ${ }^{1,2,3}$, Chengcheng $\mathrm{Fu}^{1,2,3}$, \\ Miao Miao ${ }^{1,2,3^{*}}$ and Depei $W^{1,2,3^{*}}$ \\ ${ }^{1}$ National Clinical Research Center for Hematologic Diseases, Jiangsu Institute of Hematology, The First Affiliated Hospital of \\ Soochow University, Suzhou, China, ${ }^{2}$ Institute of Blood and Marrow Transplantation, Collaborative Innovation Center of \\ Hematology, Soochow University, Suzhou, China, ${ }^{3}$ Key Laboratory of Thrombosis and Hemostasis of Ministry of Health, \\ Suzhou, China, ${ }^{4}$ Nanfang Hospital, Southern Medical University, Guangzhou, China, ${ }^{5}$ Peking University People's Hospital, \\ Peking University Institute of Hematology, National Clinical Research Center for Hematologic Disease, Research Unit of Key \\ Technique for Diagnosis and Treatments of Hematologic Malignancies, Beijing, China, ${ }^{6}$ Beijing Key Laboratory of Hematopoietic \\ Stem Cell Transplantation, Beijing, China, ${ }^{7}$ Collaborative Innovation Center of Hematology, Peking University, Beijing, China, \\ 8 Xinqiao Hospital, Army Military Medical University, Chongqing, China
}

Background: Allogeneic stem cell transplantation (allo-SCT) remains the only effective curative therapy for primary myelofibrosis. Utilization and efficacy of allo-SCT are limited by lethal complications, including engraftment failure, and acute (aGVHD) and chronic graftversus-host disease (cGVHD). Several clinical trials have explored the use of mesenchymal stem cells (MSCs) in allo-SCT to prevent hematopoietic stem cell (HSC) engraftment failure and control GVHD.

Methods: Clinical data of 17 patients with primary myelofibrosis who underwent allo-SCT combined with ex vivo expanded MSC transfusion in four centers from February 2011 to December 2018 were retrospectively analyzed.

Results: All patients received myeloablative conditioning regimen. The median number of transplanted nucleated cells (NCs) per kilogram body weight was $11.18 \times 10^{8}$ (range: 2.63-16.75 $\times 10^{8}$ ), and the median number of CD34 ${ }^{+}$cells was $4.72 \times 10^{6}$ (range: $1.32-$ $\left.8.4 \times 10^{6}\right)$. MSCs were transfused on the day of transplant or on day 7 after transplant. The median MSC infusion number was $6.5 \times 10^{6}$ (range: $0.011-65 \times 10^{6}$ ). None of the patients experienced primary or secondary graft failure in the study. The median time to neutrophil engraftment was 13 days (range: 11-22 days), and the median time to platelet engraftment was 21 days (range: 12-184 days). The median follow-up time was 40.3 months (range: 1.8-127.8 months). The estimated relapse-free survival (RFS) at 5 years was $79.1 \%$, and overall survival (OS) at 5 years was $64.7 \%$. Analysis showed that the cumulative incidence of aGVHD grade II to IV was 36\% (95\% Cl: 8\%-55\%) and that of 
grade III to IV was $26 \%$ (95\% Cl: $0 \%-45 \%$ ) at day 100 . The cumulative incidence of overall cGVHD at 2 years for the entire study population was $63 \%$ (95\% Cl: $26 \%-81 \%)$. The cumulative incidence of moderate to severe cGVHD at 2 years was 17\% (95\% Cl: 0\%42\%). Seven patients died during the study, with 5 patients succumbing from non-relapse causes and 2 from disease relapse.

Conclusion: The findings of the study indicate that allo-SCT combined with MSC transfusion may represent an effective treatment option for primary myelofibrosis.

Keywords: primary myelofibrosis, allogeneic stem cell transplantation, mesenchymal stem cells, graft failure, overall survival

\section{INTRODUCTION}

Primary myelofibrosis (PMF) is a myeloproliferative neoplasm (MPN) characterized by abnormal proliferation of megakaryocytes, bone marrow (BM) fibrosis, and extramedullary hematopoiesis. Patients with symptomatic PMF have less than 5 years' median survival (1). Conventional therapies and Janus kinases (JAK) inhibitors are mainly used for palliative purposes and have not been demonstrated to favorably modify disease natural history or prolong patient survival (2). Currently, allogeneic stem cell transplantation (allo-SCT) is the only treatment modality for PMF, and it results in disease remission and restoration of normal hematopoiesis (3).

However, despite significant advances in allo-SCT in MF, it is currently associated with a relatively high rate (range from $24 \%$ to $50 \%$ at 5 years) of transplant-related deaths or severe morbidity related to graft failure (GF), conditioning regimenrelated toxicity, lethal organ injury, infectious complications, and graft-versus-host disease (GVHD) (4-7). Although mortality from conditioning toxicity has been reduced in recent years, studies should explore methods to alleviate GF and GVHD in patients who have undergone allo-SCT. Engraftment and/or graft function can be challenging in patients with PMF undergoing an allo-SCT. GF was reported in $11 \%$ of PMF patients that is independently associated with increased mortality in patients who have undergone allo-SCT [hazard ratio (HR): 2.30] (8). In addition, GVHD is another major cause of morbidity and mortality in allo-SCT patients with PMF. The estimated 5-year survival rate of patients who develop GF and grade III-IV aGVHD was $14 \%$ and $26 \%$, respectively (8). Therefore, enhancing engraftment as well as decreasing GVHD is a potential strategy for improving efficacy of allo-SCT treatment.

Mesenchymal stem cells (MSCs) are multipotent BM cells capable of regenerating rudimentary bone in vivo and supporting hematopoiesis (9). Preclinical animal studies reported improved engraftment of hematopoietic stem cells (HSCs) and decreased risk of GVHD after co-transplantation of MSCs and HSCs (1012). These results laid a basis for further investigations on clinical application of MSC therapy to improve allo-SCT outcomes in patients with hematological disorders. Currently, MSCs are widely applied in the treatment of hematological disorders. For instance, MSCs have been used in the treatment of aplastic anemia for promoting engraftment. In addition, MSCs are also employed in treating engraftment failure and preventing and alleviating GVHD (13-15). Several clinical studies reported that cotransplantation of autologous or allogeneic MSCs with HSCs promotes BM engraftment (16-18).

To the best of our knowledge, no previous study has explored the outcomes of allo-SCT combined with MSC transfusion for patients with PMF. Therefore, there is a need to evaluate the effect of MSC infusion in combination with allo-SCT in patients with PMF. The current study explored the effects and safety of a single dose of MSC infusion during transplantation procedure in combination with myeloablative conditioning (MAC) regimen as a new allo-SCT modality for patients with PMF attending four independent centers from February 2011 to December 2018.

\section{METHODS}

\section{Eligible Patients}

The current study enrolled patients diagnosed with PMF who underwent a first allogeneic BM or peripheral blood stem cell (PBSC) transplant, with MSC transfusion prior to engraftment, admitted at 4 centers between 2011 and 2018. All patients were aged 18-65 years. In addition, patients with PMF progressing to acute leukemia were included in the current study. Patients diagnosed with myelofibrosis originating from polycythemia vera or essential thrombocythemia were excluded from the study. Data on 17 allo-SCTs were retrospectively included in the current study. All patients and donors or their legal guardians provided written informed consent prior to inclusion to the study in accordance with the Declaration of Helsinki.

\section{Conditioning Regimen and Supportive Care}

All patients received a MAC busulfan (BU)/cyclophosphamide (CY)-based regimen. Busulfan $9.6 \mathrm{mg} / \mathrm{kg}$ was intravenously administered to patients in 12 doses on days -7 to -5 . Sodium valproate was administered for busulfan-induced seizure prophylaxis. Two doses of CY $\left(3.6 \mathrm{~g} / \mathrm{m}^{2}\right)$ was intravenously administered on days -4 to -3 . Mesna was administered to lower the risk of hemorrhagic cystitis. Granulocyte colony- 
stimulating factor (G-CSF) was administered subcutaneously at a dose of $5-10 \mu \mathrm{g} / \mathrm{kg}$ from day +7 after allogeneic transplantation and was continued until sustained neutrophil engraftment.

Patients with HLA-matched related donors (MRDs) received GVHD prophylaxis comprising cyclosporine A (CsA) and shortterm methotrexate (MTX). Notably, one patient received additional antithymocyte globulin (ATG) and mycophenolate mofetil (MMF) owing to old donor age. ATG, CsA, MMF, and MTX were included for GVHD prophylaxis for HLA-matched unrelated donors (MUDs) and haploidentical donors (HIDs). CsA dose was adjusted to $200-300 \mathrm{ng} / \mathrm{ml}$ serum levels and was gradually reduced and withdrawn completely on month 9 after transplantation when no GVHD was observed. MTX was administered at $15 \mathrm{mg} / \mathrm{m}^{2} /$ day on day +1 and at $10 \mathrm{mg} / \mathrm{m}^{2} /$ day on days $+3,+6$, and +11 (only for MUDs and HIDs). Here, 15 $\mathrm{mg} / \mathrm{kg}$ MMF was administered orally twice a day from days -9 to +30 and then gradually decreased by day +40 when no GVHD was observed. ATG was administered on days -5 to -2 at a total dose of $10 \mathrm{mg} / \mathrm{kg}$ in haploidentical allo-SCT setting (19).

\section{Graft Collection and Infusion}

Stem cell mobilization of donors was performed by subcutaneous injection of 5 consecutive days of recombinant human G-CSF (rhG-CSF) at a dose of $10 \mu \mathrm{g} / \mathrm{kg} /$ day. The first day of stem cell infusion was denoted as "day 01 ", and the second day of infusion was denoted a "day 02". For MRDs and HIDs, graft source was BM combined with PBSCs. BM was collected on day 01 via BM aspiration in the operating room. PBSCs were collected on the following day (day 02) by apheresis using a COBE Spectra device (Gambro BCT, Lakewood, CO, USA). For MUDs, PBSCs were the sole graft source and were collected on day 01 . Target nucleated cell (NC) count was expected to achieve $6-10 \times 10^{8} / \mathrm{kg}$ of recipient weight, minimum $2 \times 10^{8} / \mathrm{kg}$ under specific occasions. If the target count of cells was insufficient, additional PBSCs were collected on the next day. Fresh BM and PBSCs were infused into the recipient on the day of their collection.

\section{Definitions and Disease Monitoring}

The first day of the absolute neutrophil count (ANC) $>0.5 \times 10^{9} / \mathrm{L}$ for 3 consecutive days was defined as neutrophil engraftment. The first day when the platelet count was $>20 \times 10^{9} / \mathrm{L}$ without transfusion support for 7 consecutive days was defined as platelet engraftment. Primary GF was defined as failure to achieve neutrophil engraftment within the first 28 days after stem cell infusion documentation of autologous reconstitution by chimerism analysis in the absence of relapse. Secondary GF was defined as recurrent $\mathrm{ANC} \leq 0.5 \times 10^{9} / \mathrm{L}$ after initial engraftment in the absence of reversible causes of drop in counts. Relapse was defined as reappearance or persistence of host cells with pretransplant morphological, cytogenetic, or molecular markers of the disease. Acute GVHD was scored based on a criteria proposed by the 1994 Consensus Conference on Acute GVHD Grading (20). cGVHD was evaluated based on the NIH Consensus Development Project on Criteria for Clinical Trials in Chronic Graft-versus-Host Disease: Diagnosis and Staging Working Report (21). Early mortality referred to death within
60 days after allo-SCT. Polymerase chain reaction was performed to explore donor chimerism weekly from the time of neutrophil recovery. All patients underwent BM examination after allo-SCT monthly during the first 3 months and every 3-6 months during the following 1-2 years in order to evaluate marrow cellularity and disease status.

\section{Preparation of Mesenchymal Stem Cells}

MSCs were ex vivo expanded and derived from donor or relative BM in 11 patients. BM mononuclear cells were separated by Lymphoprep (Axis-Shield, Oslo, Norway) density gradient centrifugation as previously described (15). Washed BM mononuclear cells were resuspended in $\alpha$ modified Eagle's medium (Gibco, Shanghai, China) supplemented with 10\% fetal bovine serum (Gibco, Shanghai, China) and cultured with a density of $1.0 \times 10^{6}$ cells per $\mathrm{cm}^{2}$. Cultures were maintained at $37^{\circ} \mathrm{C}$ in a humidified atmosphere containing $5 \% \mathrm{CO}_{2}$ in $15-\mathrm{cm}$ culture dish (NEST, Wuxi, China). When the cultures were near confluence $(>85 \%)$, the cells were detached with $0.25 \%$ trypsin and EDTA solution (Gibco, Shanghai, China) and cultured at a density of 4,000 cells per $\mathrm{cm}^{2}$. When $5 \times 10^{6}$ cells or more were obtained, they were harvested and washed repeatedly. The cells are formulated in saline solution containing $1 \%$ human serum albumin. Criteria for release of MSCs for clinical use included spindle-shape cell, no visible cell clumps, absence of contamination by pathogens, viability greater than $92 \%$ as determined by trypan blue testing, and immune phenotyping proving expression of CD73, CD29, CD44, CD105, and CD90 surface molecules $(>95 \%)$ and negative for markers of hematopoietic lineages, CD45, CD14, CD34, and human leukocyte antigen-DR (HLA$\mathrm{DR})$ receptors $(<5 \%)$. The complete cell culture process consists of a total of 4 cell passages. All manufacturing activities were performed in strict compliance with National Medical Products Administration's good manufacturing practice (GMP) standards. In addition, MSCs were obtained from third-party BM source in four patients as previously reported (22). MSCs were obtained from third-party umbilical cord (UC) blood (UCB) and thirdparty UC sources (iCELL Biotechnology) with the informed consent of the mother in the other 2 patients, as previously reported (23) The immunophenotype of UC-MSCs included positivity for CD13, CD29, CD90, CD44, CD105, and CD73 and negativity for CD14, CD34, CD38, CD45, CD31, and HLA-DR. The immunophenotype of UCB-MSCs was positive for CD29, CD44, CD73, CD90, CD105, and CD166 and negative for CD34, CD45, CD14, and HLA-DR. MSCs were administered as a single dose at the day of stem cell infusion in 5 patients, and the remaining 12 patients received MSCs on day 7 after transplant.

\section{Statistical Analysis}

Baseline characteristics and demographics were recorded. Continuous variables that did not follow normal distribution were expressed as median and range. Time to event duration was calculated in months or days from the date of transplant to the date of the event or last date of follow-up for patients who were alive throughout the study. Cumulative engraftment rate, cumulative incidence of aGVHD and cGVHD, relapse-free 
survival (RFS), and overall survival (OS) were explored using Kaplan-Meier estimator. Survival and cumulative incidence estimate at the specified time points also report numbers of patients at risk and two-sided 95\% confidence intervals (CIs). Death without aGVHD/cGVHD and relapse was the competing event for estimating GVHD incidence and RFS. Statistical analyses were performed using RStudio ( $\mathrm{R}$ version 3.6.1).

\section{RESULTS}

\section{Patient-, Disease-, and Transplantation- Related Characteristics}

Characteristics of included patients are presented in Table $\mathbf{1}$. Adult patients (10 males, 7 females) with a median age of 41 years (range: 22-55 years) at diagnosis were included in the current study. All patients were diagnosed with PMF. Notably, 3 patients had leukemic transformation at the time of transplant (patient No. 13, 14, 16). Disease history varied widely different, with diagnosis-to-transplant time ranging from 2 months to 10 years (median: 18 months). Gene mutations were negative in five patients. For driver mutations, a mutation in JAK2 was confirmed in 5 cases, followed by CALR mutation in 3, and none harbored MPL mutation. Six patients harbored ASXL1 gene mutation, and four of them harbored also mutations affecting JAK2 and CALR genes. U2AF1 mutation was found in 3 patients. EZH2 was mutated in 1 and IDH1/2 in 1 who also harbored mutations in K/NRAS. One patient harbored both mutations in SF3B1 and SETBP1. All study patients were categorized according to the DIPSS plus risk category, which was defined by the International Working Group (IWG) for MF (24). Risk profile was intermediate-1 in 2 patients, intermediate2 in 2 patients, and high in 13 patients. Ten patients received ruxolitinib before transplantation, one patient had previously undergone splenectomy. Stable transfusion dependency was observed in 10 patients ( 5 out of the 10 patients were under ruxolitinib treatment). Comorbidity scores determined using the Hematopoietic Cell Transplantation Comorbidity Index (HCT$\mathrm{CI}$ ) ranged from 0 to 4 (25). Notably, 14 patients were in the $0-1$ score group, and 3 patients had HCT-CI scores from 2 to 4. Thirteen patients were classified as MF-3 based on the WHO criteria at the time of transplant (26). Severe splenomegaly defined as length of the spleen exceeding the umbilicus was observed in 7 patients. Transplant details are presented in Table 2. A total of 5 patients received the graft from MRD, 3 patients received the graft from MUD, and 9 patients received the graft from HID. The graft source was BM-derived stem cells (BMSCs) combined with mobilized PBSCs for all related donors $(\mathrm{N}=14)$. The other 3 patients received mobilized PBSCs from MUDs. Donor and recipient ABO type was compatible in most patients $(\mathrm{N}=11)$. Major incompatibility was observed in 3 patients, minor incompatibility was observed in 2 patients, and bidirectional mismatch was reported in 1 patient. The median donor age was 34 (range: $16-48$ years). Median MSC infusion dose count was $6.5 \times 10^{6}$ (range: $0.011-65 \times 10^{6}$ ).

\section{Engraftment}

Median number of transplanted NCs per kilogram body weight was $11.18 \times 10^{8}$ (range: $2.63-16.75 \times 10^{8}$ ) and that of $\mathrm{CD} 34^{+}$cells was $4.72 \times 10^{6}$ (range: $\left.1.32-8.4 \times 10^{6}\right)\left(\mathrm{CD} 34^{+}\right.$proportion was not detected in one of the patients) (Table 2). Notably, no cases of primary or secondary GF were observed in the study. All patients achieved sustained full donor chimerism in the peripheral blood except 2 relapse patients. The findings showed that the engraftment rate was $100 \%$ with a median time to neutrophil engraftment of 13 days (range: $11-22$ days). The cumulative neutrophil engraftment rate was 71\% (95\% CI: $39 \%-86 \%)$ at day +15 and $100 \%$ at day +28 . Cumulative probability of platelet engraftment was 59\% (95\% CI: $27 \%-$ $77 \%$ ) at day +28 and $88 \%$ (95\% CI: $57 \%-97 \%$ ) at day +100 (Figures 1C, D). Platelet recovery showed a median time of 21 days (range: 12-184 days). All patients presented sustained engraftment except for the two relapse patients.

\section{Graft-Versus-Host Disease}

Details on GVHD are presented in Table 2 and Figures 1E-I. Acute GVHD was observed in 8 patients, with all cases presenting in the first 100 days after transplantation. Four patients presented with grade III-IV aGVHD. Notably, 3 out of the 4 patients had received graft from an haploidentical related donor. Cumulative incidence of grade I-IV aGVHD was $47 \%$ (95\% CI: 17\%-66\%). Incidence of acute GVHD grade II-IV was $36 \%$ (95\% CI: $8 \%-55 \%$ ) and incidence of grade III-IV was $26 \%$ (95\% CI: $0 \%-45 \%)$ at day 100.

cGVHD was observed in 9 patients, with onset occurring in the first 2 years after transplantation for all cases. cGVHD was mild in 8 cases and moderate in 1 case who received the graft from a MUD. No severe cGVHD was observed. Cumulative incidence of overall cGVHD at 2 years for the entire study population was $63 \%$ (95\% CI: $26 \%-81 \%$ ). Cumulative incidence of moderate to severe cGVHD at 2 years was $17 \%$ (95\% CI: $0 \%-$ $42 \%)$. All patients had clinical response to immunosuppressive therapy, and no deaths secondary to CGVHD was reported.

\section{Transplant-Related Complications}

A total of 7 patients died during follow-up (median: 40.3 months). The death of 5 out of the 7 patients was attributed to non-relapse causes (patient nos. 5, 6, 7, 11, and 16), and 2 out of the 5 patients experienced early mortality (1.8 and 1.8 months). Causes of early death were immunologic cerebral vasculitis and cytomegalovirus (CMV) pneumonia. Notably, the patient who succumbed to immunologic cerebral vasculitis had been diagnosed with congenital abnormalities of cerebrovascular maldevelopment and had a long history of recurrent epilepsy before transplant (patient no. 11). Patient no. 6 died at 11.6 months after transplant due to heart failure that was caused by persistent atrial fibrillation onset before PMF diagnosis. Patient no. 7 died from toxic epidermal necrolysis syndrome at 6.5 months. Two patients (12\%) presented with CMV reactivation, and both developed CMV pneumonia.

Safety profile of the patients was monitored throughout the study. The findings showed that no patients presented with 
TABLE 1 | Patient- and disease-related characteristics.

\begin{tabular}{|c|c|c|c|c|c|c|c|c|c|c|c|c|c|c|c|}
\hline $\begin{array}{l}\text { Patient } \\
\text { No. }\end{array}$ & Sex & $\begin{array}{c}\text { Age, } \\
\text { y }\end{array}$ & Diagnosis & $\begin{array}{l}\text { DIPSS- } \\
\text { plus } \\
\text { risk }\end{array}$ & Cytogenetics & Mutations & $\begin{array}{l}\text { HCT- } \\
\text { Cl }\end{array}$ & ECOG & $\begin{array}{l}\text { Transfusion } \\
\text { dependency }\end{array}$ & $\begin{array}{c}\text { Time } \\
\text { diagnosis-to- } \\
\text { alloSCT, } m\end{array}$ & Splenectomy & $\begin{array}{l}\text { Pre- } \\
\text { transplant } \\
\text { ruxolitinib }\end{array}$ & $\begin{array}{l}\text { Fibrosis } \\
\text { grade } \\
\text { before } \\
\text { allo-SCT }\end{array}$ & $\begin{array}{c}\text { Severe } \\
\text { Splenomegaly }\end{array}$ & $\begin{array}{l}\text { Follow } \\
\text { up, } m\end{array}$ \\
\hline 1 & $\mathrm{~F}$ & 39 & PMF & High & 46, XX, del(18)(q12q23) & Negative & 3 & 1 & Y & 12 & $\mathrm{~N}$ & $\mathrm{~N}$ & MF-3 & $\mathrm{N}$ & 74.9 \\
\hline 2 & M & 30 & PMF & Int-1 & Normal & JAK2 & 1 & 1 & $\mathrm{~N}$ & 12 & $\mathrm{~N}$ & Y & MF-2 & $\mathrm{N}$ & 64.3 \\
\hline 3 & M & 45 & PMF & High & $\begin{array}{l}48, X,-Y, ? 1 q^{-}, \operatorname{der}(3), t(1 ; 3) \\
\text { (q11;p24), der(4), t(4);? } \\
\text { (p15);?[2]/46, XY[8] }\end{array}$ & CALR & 4 & 2 & Y & 120 & $N$ & Y & MF-3 & $\mathrm{N}$ & 56.8 \\
\hline 4 & M & 47 & PMF & High & 47, idem, +21 & JAK2, ASXL1 & 3 & 2 & Y & 4 & $\mathrm{~N}$ & $\mathrm{Y}$ & MF-2 & $\mathrm{Y}$ & 50.5 \\
\hline 5 & M & 35 & PMF & Int-1 & Normal & CALR & 0 & 1 & N & 12 & N & Y & MF-3 & Y & 2.1 \\
\hline 6 & $\mathrm{~F}$ & 49 & PMF & Int-2 & Normal & Negative & 1 & 1 & Y & 36 & $\mathrm{~N}$ & $\mathrm{~N}$ & MF-2 & $\mathrm{N}$ & 11.6 \\
\hline 7 & M & 52 & PMF & High & $48, X Y,+8$ & JAK2, ASXL1 & 0 & 1 & $N$ & 42 & $N$ & Y & MF-3 & $\mathrm{N}$ & 6.5 \\
\hline 8 & $\mathrm{~F}$ & 25 & PMF & Int-2 & Normal & Negative & 0 & 1 & $\mathrm{~N}$ & 24 & $\mathrm{~N}$ & $\mathrm{Y}$ & MF-3 & $\mathrm{Y}$ & 31.7 \\
\hline 9 & M & 42 & PMF & High & Normal & ASXL1 & 1 & 1 & Y & 2 & N & $\mathrm{N}$ & MF-3 & $\mathrm{N}$ & 71.3 \\
\hline 10 & $\mathrm{~F}$ & 31 & PMF & High & $47, X X,+8$ & U2AF1, SETD2 & 0 & 2 & Y & 2 & $N$ & $N$ & MF-3 & $N$ & 54.7 \\
\hline 11 & M & 25 & PMF & High & $47, X Y,+8$ & U2AF1, ASXL1 & 0 & 2 & $\mathrm{~N}$ & 24 & $\mathrm{~N}$ & $\mathrm{~N}$ & MF-2 & Y & 1.8 \\
\hline 12 & $\mathrm{~F}$ & 51 & PMF & High & Normal & Negative & 1 & 2 & Y & 36 & $\mathrm{~N}$ & N & MF-3 & $\mathrm{N}$ & 77.4 \\
\hline 13 & M & 41 & PMF & High & $45, X Y,-9,+10,-7,5 q-$ & $\begin{array}{l}\text { IDH1, IDH2, } \\
\text { NRAS, JAK2, } \\
\text { KRAS, U2AF1, } \\
\text { ASXL1 }\end{array}$ & 0 & 0 & Y & 18 & Y & Y & MF-3 & Y & 32.9 \\
\hline 14 & M & 51 & PMF & High & $47, X Y,+8, \operatorname{inv}(9)(p 12 q 13)$ & $\begin{array}{l}\text { RUNX1, CALR, } \\
\text { EZH2, ASXL1 }\end{array}$ & 0 & 0 & N & 42 & $N$ & Y & MF-3 & N & 19.7 \\
\hline 15 & $\mathrm{~F}$ & 41 & PMF & High & $46, X X$, del(20)(q11) & SF3B1, SETBP1 & 0 & 0 & Y & 11 & $\mathrm{~N}$ & Y & MF-3 & Y & 40.3 \\
\hline 16 & $\mathrm{~F}$ & 55 & PMF & High & $45, X X, t(3,3)(q 21: q 26),-7$ & $\begin{array}{l}\text { JAK2-V617F, } \\
\text { EVI1, TET2, } \\
\text { HOX11 }\end{array}$ & 1 & 1 & Y & 8.7 & $N$ & Y & MF-3 & Y & 1.8 \\
\hline 17 & M & 22 & PMF & High & Normal & Negative & 0 & 2 & $\mathrm{~N}$ & 48 & $\mathrm{~N}$ & $\mathrm{~N}$ & MF-3 & N & 127.8 \\
\hline
\end{tabular}

F, female; M, male; PMF, primary myelofibrosis; Int-1, intermediate-1; Int-2, intermediate-2; HCT-Cl, hematopoietic cell transplantation-comorbidity index; ECOG, Eastern Cooperative Oncology Group score; Y, yes; N, no. 
TABLE 2 | Transplantation-related characteristics.

\begin{tabular}{|c|c|c|c|c|c|c|c|c|c|c|c|c|c|c|c|}
\hline $\begin{array}{l}\text { Patient } \\
\text { No. }\end{array}$ & $\begin{array}{c}\text { GVHD } \\
\text { prophylaxis }\end{array}$ & $\begin{array}{l}\text { Donor } \\
\text { type }\end{array}$ & $\begin{array}{l}\text { Donor/ } \\
\text { recipient } \\
\text { ABO type }\end{array}$ & $\begin{array}{l}\text { Donor } \\
\text { age }\end{array}$ & $\begin{array}{l}\text { Donor } \\
\text { sex }\end{array}$ & $\begin{array}{l}\text { NCs dose } \\
\text { count, } \\
\times 10^{8} / \mathrm{kg}\end{array}$ & $\begin{array}{l}\text { CD34 } 4^{+} \text {cell } \\
\text { dose count, } \\
\times 10^{6} / \mathrm{kg}\end{array}$ & $\begin{array}{c}\text { MSCs } \\
\text { dose } \\
\text { count, } \\
\times 10^{6}\end{array}$ & $\begin{array}{l}\text { Time to neu- } \\
\text { trophil } \\
\text { engraftment }\end{array}$ & $\begin{array}{c}\text { Time to } \\
\text { platelet } \\
\text { engraftment }\end{array}$ & $\begin{array}{c}\text { aGVHD } \\
\text { grade }\end{array}$ & $\begin{array}{l}\text { cGVHD } \\
\text { grade }\end{array}$ & Complications & $\begin{array}{l}\text { Current } \\
\text { status }\end{array}$ & $\begin{array}{c}\text { Cause of } \\
\text { death }\end{array}$ \\
\hline 1 & CsA+MTX & MRD & $\mathrm{AB}+/ \mathrm{A}+$ & 42 & $\mathrm{~F}$ & 11.3 & 5.23 & 6.5 & 11 & 15 & / & Mild & / & Alive & / \\
\hline 2 & $\begin{array}{l}\text { ATG+MMF } \\
+\mathrm{CsA}+\mathrm{MTX}\end{array}$ & MUD & $\mathrm{A}+/ \mathrm{A}+$ & 45 & $\mathrm{~F}$ & 2.63 & 5 & 6.5 & 13 & 17 & / & / & / & Alive & / \\
\hline 3 & $\begin{array}{l}\text { ATG+MMF } \\
+\mathrm{CsA}+\mathrm{MTX}\end{array}$ & HID & $\mathrm{A}+/ \mathrm{A}+$ & 26 & M & 12.72 & 6.31 & 6.7 & 17 & 21 & 1 & Mild & / & Alive & / \\
\hline 4 & $\begin{array}{l}\text { ATG+MMF } \\
+\mathrm{CsA}+\mathrm{MTX}\end{array}$ & HID & $\mathrm{A}+/ \mathrm{O}+$ & 26 & M & 4.94 & 7.8 & 6.5 & 20 & 63 & III & l & / & Alive & / \\
\hline 5 & $\begin{array}{l}\text { ATG+MMF } \\
+\mathrm{CsA}+\mathrm{MTX}\end{array}$ & HID & $\mathrm{O}+/ \mathrm{O}+$ & 34 & $\mathrm{~F}$ & 16.67 & 4.84 & 6.5 & 13 & 26 & / & / & TMA & $\begin{array}{l}\text { Died on } \\
\text { day } 64\end{array}$ & TMA \\
\hline 6 & $\begin{array}{l}\text { ATG+MMF } \\
+\mathrm{CsA}+\mathrm{MTX}\end{array}$ & HID & $\mathrm{A}+/ \mathrm{A}+$ & 45 & M & 16.75 & 5.36 & 6 & 12 & 15 & IV & Mild & $\begin{array}{l}\text { Heart failure, } \\
\text { cystitis }\end{array}$ & $\begin{array}{l}\text { Died on } \\
\text { day } 349\end{array}$ & Heart failure \\
\hline 7 & $\begin{array}{l}\text { ATG+MMF } \\
+\mathrm{CsA}+\mathrm{MTX}\end{array}$ & HID & $\mathrm{O}+/ \mathrm{O}+$ & 20 & M & 14.44 & 4.47 & 6.5 & 12 & 18 & $\|$ & Mild & Pneumonia & $\begin{array}{l}\text { Died on } \\
\text { day } 195\end{array}$ & $\begin{array}{l}\text { Toxic epiderma } \\
\text { necrolysis } \\
\text { syndrome }\end{array}$ \\
\hline 8 & $\begin{array}{l}\text { ATG+MMF } \\
+\mathrm{CsA}+\mathrm{MTX}\end{array}$ & MUD & $\mathrm{O}+/ \mathrm{A}+$ & 24 & $\mathrm{~F}$ & 6.72 & 4.5 & 6 & 11 & 60 & / & / & $\begin{array}{l}\text { CMV/EBV } \\
\text { pneumonia }\end{array}$ & Alive & i \\
\hline 9 & CsA+MTX & MRD & $\mathrm{A}+/ \mathrm{A}+$ & 48 & $\mathrm{~F}$ & 12.27 & 4.2 & 6.5 & 11 & 12 & I & Mild & / & $\begin{array}{l}\text { Died on } \\
\text { day } \\
2138\end{array}$ & Relapse \\
\hline 10 & CsA+MTX & MRD & $\mathrm{O}+/ \mathrm{O}+$ & 30 & $\mathrm{~F}$ & 15.03 & 4.59 & 60 & 13 & 30 & / & Mild & / & Alive & / \\
\hline 11 & $\begin{array}{l}\text { ATG+MMF } \\
+ \text { CsA+MTX }\end{array}$ & HID & $\mathrm{AB}+/ \mathrm{B}+$ & 47 & M & 11.18 & 5.42 & 65 & 11 & 17 & $\|$ & / & $\begin{array}{l}\text { Immunologic } \\
\text { cerebral vasculitis, } \\
\text { pneumonia }\end{array}$ & $\begin{array}{l}\text { Died on } \\
\text { day } 53\end{array}$ & $\begin{array}{l}\text { Immunologic } \\
\text { cerebral } \\
\text { vasculitis }\end{array}$ \\
\hline 12 & $\begin{array}{l}\text { ATG+MMF } \\
+\mathrm{CsA}+\mathrm{MTX}\end{array}$ & MUD & $\mathrm{A}+\mathrm{B}+$ & 20 & M & 6.4 & 8.4 & 50 & 22 & 184 & / & Moderate & / & Alive & 1 \\
\hline 13 & $\begin{array}{l}\text { ATG+MMF } \\
+\mathrm{CsA}+\mathrm{MTX}\end{array}$ & HID & $\mathrm{O}+/ \mathrm{B}+$ & 16 & M & 10.69 & 3.18 & 0.011 & 11 & 13 & III & 1 & 1 & Alive & 1 \\
\hline 14 & $\begin{array}{l}\text { ATG+MMF } \\
+\mathrm{CsA}+\mathrm{MTX}\end{array}$ & MRD & $\mathrm{O}+/ \mathrm{O}+$ & 42 & $\mathrm{~F}$ & 10.47 & 3.56 & 0.011 & 12 & 19 & / & Mild & / & $\begin{array}{l}\text { Died on } \\
\text { day } 590\end{array}$ & Relapse \\
\hline 15 & CsA+MTX & MRD & $\mathrm{O}+/ \mathrm{O}+$ & 35 & $\mathrm{~F}$ & 10.47 & 1.72 & 0.011 & 13 & 32 & III & Mild & 1 & Alive & / \\
\hline 16 & $\begin{array}{l}\text { ATG+MMF } \\
+\mathrm{CsA}+\mathrm{MTX}\end{array}$ & HID & $\mathrm{B}+/ \mathrm{B}+$ & 27 & $\mathrm{~F}$ & 6.58 & 1.32 & 0.011 & 18 & 37 & / & / & $\begin{array}{l}\mathrm{CMV} \text { reactivation, } \\
\text { cystitis }\end{array}$ & $\begin{array}{l}\text { Died on } \\
\text { day } 55\end{array}$ & $\begin{array}{l}\text { CMV } \\
\text { pneumonia }\end{array}$ \\
\hline 17 & $\begin{array}{l}\text { ATG+MMF } \\
+\mathrm{CsA}+\mathrm{MTX}\end{array}$ & HID & $\mathrm{A}+/ \mathrm{A}+$ & 46 & M & 11.4 & / & 60 & 16 & 103 & / & / & $\begin{array}{l}\text { Pneumonia, drug- } \\
\text { induced liver injury }\end{array}$ & Alive & / \\
\hline
\end{tabular}

GVHD, graft-versus-host disease; CSA, cyclosporine A; ATG, antithymocyte immunoglobulin; MMF, mycophenolate mofetil; MTX, methotrexate; $F$, female; $M$, male; MRD, matched related donor; MUD, matched unrelated donor; HID, haploidentical donor; NCs, nucleated cells; MSCs, mesenchymal stem cells; CMV, cytomegalovirus; EBV, Epstein-Barr virus; "/", not occurred; TMA, thrombotic microangiopathy. 
A

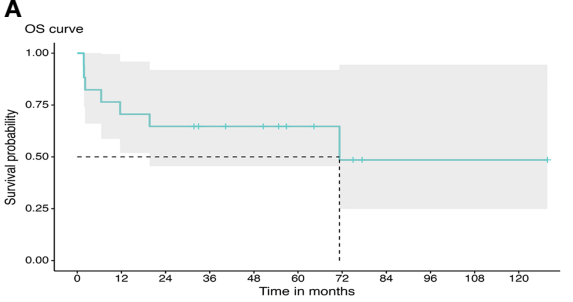

Number at risk: $\mathrm{n}(\%)$

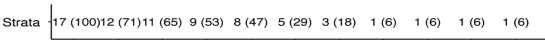

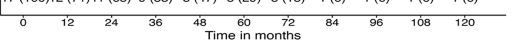

C

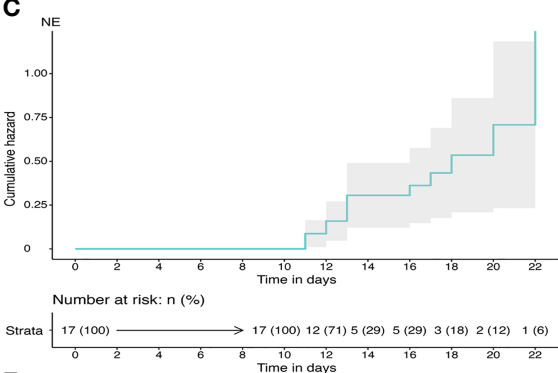

E

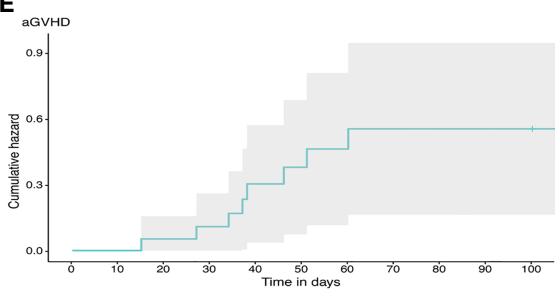

Number at risk: $\mathrm{n}(\%)$

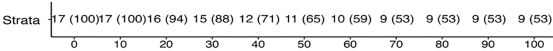

G

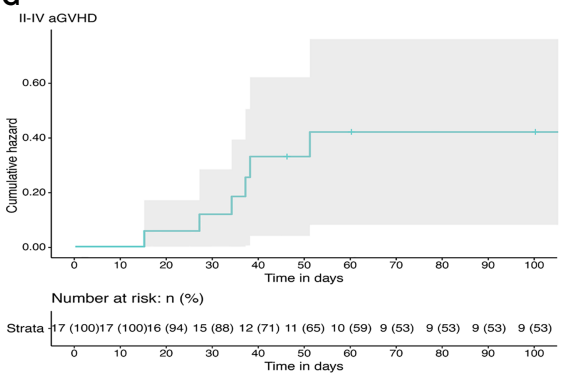

$I_{\text {M/s cGVHD }}$

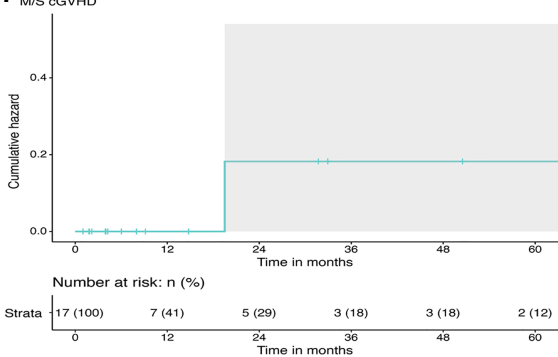

B

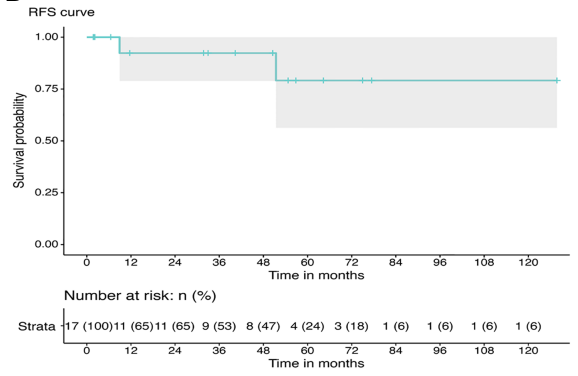

D

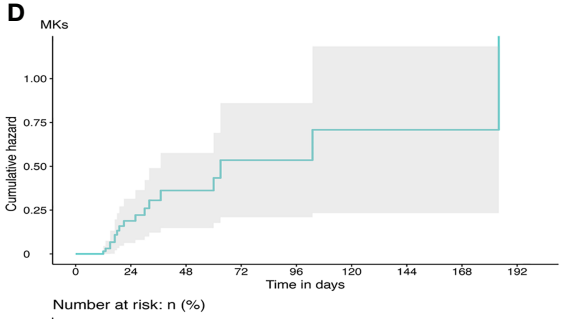

Strata \begin{tabular}{ccccccccc}
$17(100)$ & $8(47)$ & $4(24)$ & $2(12)$ & $2(12)$ & $1(6)$ & $1(6)$ & $1(6)$ & $0(0)$ \\
\hline 0 & 24 & 48 & 72 & 96 & 120 & 144 & 168 & 192
\end{tabular}

$\mathbf{F}$

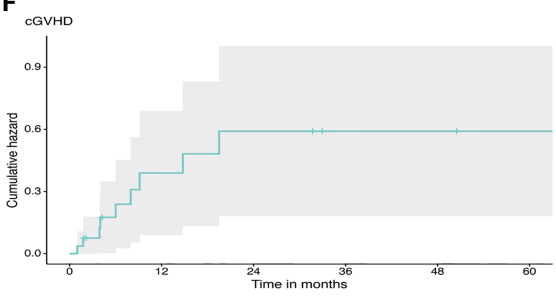

Number at risk: $\mathrm{n}(\%)$

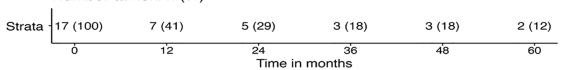

H

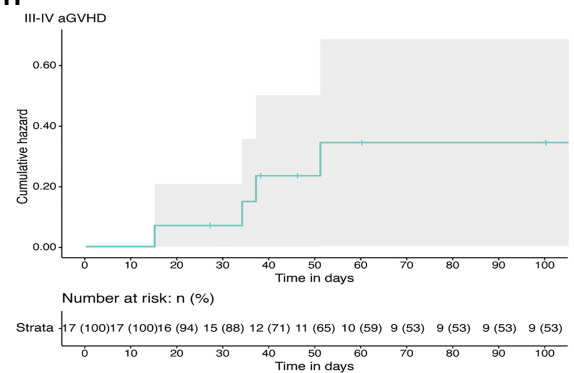

FIGURE 1 | Transplant outcomes in primary myelofibrosis. (A) The probability of OS. (B) The probability of RFS. (C) The cumulative incidence of neutrophil engraftment. (D) The cumulative incidence of platelet engraftment. (E) The total cumulative incidence of aGVHD. (F) The total cumulative incidence of cGVHD. (G) The cumulative incidence of grade II-IV aGVHD. (H) The cumulative incidence of grade III-IV aGVHD. (I) The cumulative incidence of moderate to severe cGVHD. OS, overall survival; RFS, relapse-free survival; NE, neutrophil; MK, megakaryocyte; aCVHD, acute graft-versus-host disease; cGVHD, chronic graft-versus-host disease. 
allergic hypersensitivity, fever, hypertension, pulmonary embolism, or other serious complications directly related to MSC infusion.

\section{Relapse and Survival Rates}

Disease relapse was observed in 2 cases at 51.4 and 9 months after transplantation (patient no. 9 and patient no. 14, respectively). The two patients harbored mutations in ASXL1 gene and received stem cells from MRD. The patients died from disease progression without undergoing a second transplant. Outcome information of patients is presented in Table 2 and Figures 1A, B. Estimated RFS at 5 years was 79\% (95\% CI: 56\%$100 \%)$. Patients estimated that OS at 5 years was 65\% (95\% CI: $46 \%-92 \%$ ). Median follow-up duration was 40.3 months (range: 1.8-127.8 months).

\section{DISCUSSION}

Allo-SCT is the only treatment modality for curing PMF. Despite the benefit, the associated comorbidities coupled with relatively poor engraftment limit extensive utilization of allo-SCT even in symptomatic PMF patients $(4,27)$. Thus, a supplementary remedy to boost BM engraftment should be developed to enhance clinical outcomes after allo-SCT. The current study explored whether MSC transfusion can improve transplantation outcomes (engraftment, GVHD, and relapse) in an allo-SCT setting.

GF continues to remain a major barrier of allo-HCT in MF patients, and rates varying from $2 \%$ to as high as $28 \%$ have been reported in different settings $(6,27)$. Coupled with GF, delayed hematopoietic reconstitution was also observed. CIBMTR data previously reported a cumulative incidence of neutrophil engraftment of $84 \%$ at 28 days and $97 \%$ at 100 days. The probability of platelet engraftment was significantly lower with $47 \%$ at 28 days and $77 \%$ at 100 days (5). In the current study, all patients achieved successful engraftment and faster hematopoietic reconstitution with $100 \%$ neutrophil engraftment at 28 days and $59 \%$ platelet engraftment incidence at 28 days and $88 \%$ at 100 days. Addition of MSC grafts may be beneficial to improve engraftment. This finding aligned with that reported by Liu et al. (28) that MSC coinfusion improved platelet recovery. In another pediatric study, Ball et al. (17) reported similar findings that cotransplanted donor BM-derived MSCs with HSCs resulted in sustained hematopoietic engraftment. Studies report that infusion of MSCs is effective in the treatment of many diseases such as ischemic stroke, diabetes mellitus type I, and hematological diseases owing to their immunomodulatory, angiogenic, antiapoptotic, and antifibrotic therapeutic activities and their ability to support stem cells (29, 30). In addition, MSCs have been utilized as an adjuvant cellular therapy to promote rapid hematopoietic reconstitution in allo-SCT patients by inhibiting apoptosis and differentiation of HSCs and inducing engraftment of precursor cells in the BM niche $(31,32)$. Mounting evidence has identified that most clinical effects exhibited by MSCs are mainly linked to their paracrine effects (33). MSCs produce factors such as CXCL12 stem cell factor (SCF) that can help in recruiting HSCs and supporting hematopoiesis (34). MSC conditioned medium administration also seemed to enhance BM engraftment in part by restoring vasculature via pleiotrophin production (35). Moreover, the type of conditioning (RIC vs. MAC or non-MAC) might also affect the occurrence of GF (27). A reduced-intensity conditioning (RIC) trial comparing fludarabine in combination with busulfan or thiotepa with MF patients confirmed a GF rate of $14 \%$ vs. $10 \%$ (36). Relatively high engraftment in the current study can be partly attributed to MAC regimen with busulfan that lowers the risk of rejection (37).

GVHD represents another major challenge that results in lifethreatening complications after allo-SCT in PMF patients. Although unidentified, in theory, the inflammatory cytokines that mediate aGVHD are also implicated in mediation of the constitutional symptoms in PMF (38). Moreover, a relatively higher number of transfused cells may also contribute to a higher incidence of GVHD in PMF patients. By inhibiting the activation and proliferation of activated $\mathrm{T}$ cells, limiting tissue damage, and promoting tissue regeneration, application of MSCs in aGVHD treatment has achieved great success $(39,40)$. Distinguished from the treatment of GVHD, whether infusion of MSCs can prevent GVHD remains inconclusive $(32,41)$. In our study, MSCs did not show a significant effect on the incidence of GVHD, as indicated by the incidence of $36 \%$ in grade II-IV aGVHD and $63 \%$ incidence of cGVHD by 2 years. This finding is consistent with results from Liu et al. (28) and Lee et al. (42), indicating that coinfusion of MSC had no effect on GVHD incidence. Similarly, the incidence of GVHD in the current study is comparable to that of a non-MSC-based allo-SCT trial with MF patients (6). This result may be attributed to the relatively lower dose and single infusion of MSCs compared with a previous report that indicated that MSCs preferably be administered at an average dose of $1.0 \times 10^{6} / \mathrm{kg}(43)$. The observed slightly increased incidence of GVHD in the entire group can be attributed to the low cases of MRD transplant.

Some studies reported a higher incidence of CMV/EBV reactivation and disease recurrence owing to immunosuppressive effects of MSCs $(44,45)$. Nevertheless, it is still a topic of debate. Other studies report that MSC transfusion does not increase the incidence of infection or disease recurrence during transplant or for aGVHD treatment $(17,43,46)$. In the current study, neither the reactivation of CMV/EBV nor disease relapse was worsened by MSC transfusion, which was in line with previous studies. This may partly be due to the in vivo activity of MSCs that lasts 2 weeks with no significant effect on long-term infection and disease relapse. Out of the 5 patients who died from non-relapse reasons, 3 presented with severe splenomegaly and grade 3 fibrosis, and all 5 patients received graft from an HID. A study based on data from the European Society for Blood and Marrow Transplantation (EBMT) recruiting $103 \mathrm{MF}$ allo-SCT patients reported that HLAmismatch is a major risk factor for transplant-related mortality (6). A previous study recruiting mostly MAC transplants (83\%) reported a 5 -year OS of $30 \%-40 \%$ depending on donor sources (4). The 5-year OS was reported as $53.0 \%$ in the MAC cohort according to a large EBMT study (7). The estimated survival rates in the current study for RFS and OS at 5 years were $79.1 \%$ and $64.7 \%$ with a median follow-up of 40.3 months. The relatively higher OS can be partly attributed to the younger 
median age (41 years) of patients involved in the current study. Moreover, the higher survival rate compared with that of highrisk and young patients with MF who did not undergo allo-SCT corroborated potential curative effect of allo-SCT for PMF patients even with leukemia transformation (47).

This is the first report on allo-SCT combined with MSC transfusion in patients affecting PMF. Despite lack of a control arm, the present results showed promising amelioration of engraftment and increase in survival. These findings indicate that allo-SCT combined with MSC transfusion might be an attractive approach for treatment of PMF patients. Limitations of our study are those inherent to multiple center-based retrospective analyses including a lack of clarity for physician choice of time and dose of MSC infusion and heterogeneous data with GVHD prophylaxis and therapies associated with supportive care. MSC dose varied greatly in previous literature (41). Although it was reported that MSCs exerted an effect in a dose-dependent manner regarding engraftment promotion (48), the impact of MSC dose on engraftment still remains to be determined. Whereas GVHD prevention was reported not correlated with MSC dose (45). Concordantly, optimal timing of MSC infusion is inconclusive and may depend on the infusion purpose $(17,43,49)$. Further prospective, controlled, large-scale studies should be conducted to validate the finding obtained in the current study.

\section{DATA AVAILABILITY STATEMENT}

The original contributions presented in the study are included in the article/supplementary material. Further inquiries can be directed to the corresponding authors.

\section{ETHICS STATEMENT}

The studies involving human participants were reviewed and approved by the ethics committee of all the four centers involved

\section{REFERENCES}

1. Cervantes F, Dupriez B, Pereira A, Passamonti F, Reilly JT, Morra E, et al. New Prognostic Scoring System for Primary Myelofibrosis Based on a Study of the International Working Group for Myelofibrosis Research and Treatment. Blood (2009) 113(13):2895-901. doi: 10.1182/blood-2008-07-170449

2. Tefferi A. Primary Myelofibrosis: 2021 Update on Diagnosis, RiskStratification and Management. Am J Hematol (2021) 96(1):145-62. doi: 10.1002/ajh.26050

3. Thiele J, Kvasnicka HM, Dietrich H, Stein G, Hann M, Kaminski A, et al. Dynamics of Bone Marrow Changes in Patients With Chronic Idiopathic Myelofibrosis Following Allogeneic Stem Cell Transplantation. Histol Histopathol (2005) 20(3):879-89. doi: 10.14670/hh-20.879

4. Ballen KK, Shrestha S, Sobocinski KA, Zhang MJ, Bashey A, Bolwell BJ, et al. Outcome of Transplantation for Myelofibrosis. Biol Blood Marrow Transplant (2010) 16(3):358-67. doi: 10.1016/j.bbmt.2009.10.025

5. Gupta V, Malone AK, Hari PN, Ahn KW, Hu ZH, Gale RP, et al. ReducedIntensity Hematopoietic Cell Transplantation for Patients With Primary Myelofibrosis: A Cohort Analysis From the Center for International Blood and Marrow Transplant Research. Biol Blood Marrow Transplant (2014) 20 (1):89-97. doi: 10.1016/j.bbmt.2013.10.018 in the study. The patients/participants provided their written informed consent to participate in this study.

\section{AUTHOR CONTRIBUTIONS}

QW designed the research study, collected the data, analyzed the data, and wrote the article. NX designed the research study, collected the data, and analyzed the data. YW and XiZ analyzed the data. LL contributed to the data analysis and article writing. $\mathrm{HZ}, \mathrm{HW}$, and XiaZ contributed to the data analysis. XT and CF contributed to the external validation. MM and DW contributed to the research design, data analysis, writing the article, and supervision of the study. All authors read and approved the final article.

\section{FUNDING}

This study was supported by the National Natural Science Foundation of China (81730003), National Science and Technology Major Project (2017ZX09304021), National Key R\&D Program of China (2019YFC0840604, 2017YFA0104502), Key R\&D Program of Jiangsu Province (BE2019798), Priority Academic Program Development of Jiangsu Higher Education Institutions (PAPD), Jiangsu Medical Outstanding Talents Project (JCRCA2016002), Jiangsu Provincial Key Medical Center (YXZXA2016002), and Suzhou Science and Technology Program Project (SLT201911).

\section{ACKNOWLEDGMENTS}

We thank Jian Jin, Jiali Xu, and Guanghua Chen for assistance in collecting the data. We also thank Yingying Zhai and Mengxing Xue for checking the data.

6. Kröger N, Holler E, Kobbe G, Bornhäuser M, Schwerdtfeger R, Baurmann H, et al. Allogeneic Stem Cell Transplantation After Reduced-Intensity Conditioning in Patients With Myelofibrosis: A Prospective, Multicenter Study of the Chronic Leukemia Working Party of the European Group for Blood and Marrow Transplantation. Blood (2009) 114(26):5264-70. doi: 10.1182/blood-2009-07-234880

7. McLornan D, Szydlo R, Koster L, Chalandon Y, Robin M, Wolschke C, et al. Myeloablative and Reduced-Intensity Conditioned Allogeneic Hematopoietic Stem Cell Transplantation in Myelofibrosis: A Retrospective Study by the Chronic Malignancies Working Party of the European Society for Blood and Marrow Transplantation. Biol Blood Marrow Transplant (2019) 25(11):216771. doi: 10.1016/j.bbmt.2019.06.034

8. Hernández-Boluda JC, Pereira A, Kröger N, Beelen D, Robin M, Bornhäuser $\mathrm{M}$, et al. Determinants of Survival in Myelofibrosis Patients Undergoing Allogeneic Hematopoietic Cell Transplantation. Leukemia (2021) 35(1):21524. doi: 10.1038/s41375-020-0815-Z

9. Friedenstein AJ, Petrakova KV, Kurolesova AI, Frolova GP. Heterotopic of Bone Marrow. Analysis of Precursor Cells for Osteogenic and Hematopoietic Tissues. Transplantation (1968) 6(2):230-47.

10. Polchert D, Sobinsky J, Douglas G, Kidd M, Moadsiri A, Reina E, et al. IFNGamma Activation of Mesenchymal Stem Cells for Treatment and Prevention 
of Graft Versus Host Disease. Eur J Immunol (2008) 38(6):1745-55. doi: $10.1002 /$ eji.200738129

11. Maitra B, Szekely E, Gjini K, Laughlin MJ, Dennis J, Haynesworth SE, et al. Human Mesenchymal Stem Cells Support Unrelated Donor Hematopoietic Stem Cells and Suppress T-Cell Activation. Bone Marrow Transplant (2004) 33(6):597-604. doi: 10.1038/sj.bmt. 1704400

12. Noort WA, Kruisselbrink AB, in't Anker PS, Kruger M, van Bezooijen RL, de Paus RA, et al. Mesenchymal Stem Cells Promote Engraftment of Human Umbilical Cord Blood-Derived CD34(+) Cells in NOD/SCID Mice. Exp Hematol (2002) 30(8):870-8. doi: 10.1016/s0301-472x(02)00820-2

13. Petinati N, Drize N, Sats N, Risinskaya N, Sudarikov A, Drokov M, et al. Recovery of Donor Hematopoiesis After Graft Failure and Second Hematopoietic Stem Cell Transplantation With Intraosseous Administration of Mesenchymal Stromal Cells. Stem Cells Int (2018) 2018:6495018. doi: 10.1155/2018/6495018

14. Zhao K, Liu Q. The Clinical Application of Mesenchymal Stromal Cells in Hematopoietic Stem Cell Transplantation. J Hematol Oncol (2016) 9(1):46. doi: 10.1186/s13045-016-0276-z

15. Liu X, Wu M, Peng Y, Chen X, Sun J, Huang F, et al. Improvement in Poor Graft Function After Allogeneic Hematopoietic Stem Cell Transplantation Upon Administration of Mesenchymal Stem Cells From Third-Party Donors: A Pilot Prospective Study. Cell Transplant (2014) 23(9):1087-98. doi: 10.3727/ $096368912 \times 661319$

16. Wu Y, Wang Z, Cao Y, Xu L, Li X, Liu P, et al. Cotransplantation of Haploidentical Hematopoietic and Umbilical Cord Mesenchymal Stem Cells With a Myeloablative Regimen for Refractory/Relapsed Hematologic Malignancy. Ann Hematol (2013) 92(12):1675-84. doi: 10.1007/s00277-0131831-0

17. Ball LM, Bernardo ME, Roelofs H, Lankester A, Cometa A, Egeler RM, et al. Cotransplantation of Ex Vivo Expanded Mesenchymal Stem Cells Accelerates Lymphocyte Recovery and may Reduce the Risk of Graft Failure in Haploidentical Hematopoietic Stem-Cell Transplantation. Blood (2007) 110 (7):2764-7. doi: 10.1182/blood-2007-04-087056

18. Koç ON, Gerson SL, Cooper BW, Dyhouse SM, Haynesworth SE, Caplan AI, et al. Rapid Hematopoietic Recovery After Coinfusion of Autologous-Blood Stem Cells and Culture-Expanded Marrow Mesenchymal Stem Cells in Advanced Breast Cancer Patients Receiving High-Dose Chemotherapy. J Clin Oncol (2000) 18(2):307-16. doi: 10.1200/jco.2000.18.2.307

19. Wang Y, Liu Q-F, Lin R, Yang T, Xu Y-J, Mo X-D, et al. Optimizing Antithymocyte Globulin Dosing in Haploidentical Hematopoietic Cell Transplantation: Long-Term Follow-Up of a Multicenter, Randomized Controlled Trial. Sci Bull (2021) 66(24):2498-505. doi: 10.1016/j.scib.2021.06.002

20. Przepiorka D, Weisdorf D, Martin P, Klingemann HG, Beatty P, Hows J, et al. 1994 Consensus Conference on Acute GVHD Grading. Bone Marrow Transplant (1995) 15(6):825-8.

21. Filipovich AH, Weisdorf D, Pavletic S, Socie G, Wingard JR, Lee SJ, et al. National Institutes of Health Consensus Development Project on Criteria for Clinical Trials in Chronic Graft-Versus-Host Disease: I. Diagnosis and Staging Working Group Report. Biol Blood Marrow Transplant (2005) 11(12):945-56. doi: 10.1016/j.bbmt.2005.09.004

22. Chen S, Zhao K, Lin R, Wang S, Fan Z, Huang F, et al. The Efficacy of Mesenchymal Stem Cells in Bronchiolitis Obliterans Syndrome After Allogeneic HSCT: A Multicenter Prospective Cohort Study. EBioMedicine (2019) 49:213-22. doi: 10.1016/j.ebiom.2019.09.039

23. Gao L, Zhang Y, Hu B, Liu J, Kong P, Lou S, et al. Phase II Multicenter, Randomized, Double-Blind Controlled Study of Efficacy and Safety of Umbilical Cord-Derived Mesenchymal Stromal Cells in the Prophylaxis of Chronic Graft-Versus-Host Disease After HLA-Haploidentical Stem-Cell Transplantation. J Clin Oncol (2016) 34(24):2843-50. doi: 10.1200/jco.2015.65.3642

24. Gangat N, Caramazza D, Vaidya R, George G, Begna K, Schwager S, et al. DIPSS Plus: A Refined Dynamic International Prognostic Scoring System for Primary Myelofibrosis That Incorporates Prognostic Information From Karyotype, Platelet Count, and Transfusion Status. J Clin Oncol (2011) 29 (4):392-7. doi: 10.1200/jco.2010.32.2446

25. Sorror ML, Maris MB, Storb R, Baron F, Sandmaier BM, Maloney DG, et al. Hematopoietic Cell Transplantation (HCT)-Specific Comorbidity Index: A New Tool for Risk Assessment Before Allogeneic HCT. Blood (2005) 106 (8):2912-9. doi: 10.1182/blood-2005-05-2004
26. Thiele J, Kvasnicka HM, Facchetti F, Franco V, van der Walt J, Orazi A. European Consensus on Grading Bone Marrow Fibrosis and Assessment of Cellularity. Haematologica (2005) 90(8):1128-32. doi: 10.3324/\%25x

27. Slot S, Smits K, van de Donk NW, Witte BI, Raymakers R, Janssen JJ, et al. Effect of Conditioning Regimens on Graft Failure in Myelofibrosis: A Retrospective Analysis. Bone Marrow Transplant (2015) 50(11):1424-31. doi: 10.1038/bmt.2015.172

28. Liu K, Chen Y, Zeng Y, Xu L, Liu D, Chen H, et al. Coinfusion of Mesenchymal Stromal Cells Facilitates Platelet Recovery Without Increasing Leukemia Recurrence in Haploidentical Hematopoietic Stem Cell Transplantation: A Randomized, Controlled Clinical Study. Stem Cells Dev (2011) 20(10):1679-85. doi: 10.1089/scd.2010.0447

29. Le Blanc K, Mougiakakos D. Multipotent Mesenchymal Stromal Cells and the Innate Immune System. Nat Rev Immunol (2012) 12(5):383-96. doi: 10.1038/ nri3209

30. Hematti P, Kim J, Battiwalla M. Mesenchymal Stem Cells in Hematopoietic Stem Cell Transplantation. In: Srivastava R, Shankar S, editors. Stem Cells and Human Diseases. Dordrecht: Springer Netherlands (2012). p. 101-15.

31. Batsali AK, Georgopoulou A, Mavroudi I, Matheakakis A, Pontikoglou CG, Papadaki HA. The Role of Bone Marrow Mesenchymal Stem Cell Derived Extracellular Vesicles (MSC-EVs) in Normal and Abnormal Hematopoiesis and Their Therapeutic Potential. J Clin Med (2020) 9(3):856. doi: 10.3390/ jcm9030856

32. Kuzmina LA, Petinati NA, Parovichnikova EN, Lubimova LS, Gribanova EO, Gaponova TV, et al. Multipotent Mesenchymal Stromal Cells for the Prophylaxis of Acute Graft-Versus-Host Disease-A Phase II Study. Stem Cells Int (2012) 2012:968213. doi: 10.1155/2012/968213

33. Liang X, Ding Y, Zhang Y, Tse HF, Lian Q. Paracrine Mechanisms of Mesenchymal Stem Cell-Based Therapy: Current Status and Perspectives. Cell Transplant (2014) 23(9):1045-59. doi: 10.3727/096368913x667709

34. Omatsu Y, Sugiyama T, Kohara H, Kondoh G, Fujii N, Kohno K, et al. The Essential Functions of Adipo-Osteogenic Progenitors as the Hematopoietic Stem and Progenitor Cell Niche. Immunity (2010) 33(3):387-99. doi: 10.1016/ j.immuni.2010.08.017

35. Kim YH, Cho KA, Lee HJ, Park M, Shin SJ, Park JW, et al. Conditioned Medium From Human Tonsil-Derived Mesenchymal Stem Cells Enhances Bone Marrow Engraftment via Endothelial Cell Restoration by Pleiotrophin. Cells (2020) 9(1):221. doi: 10.3390/cells9010221

36. Patriarca F, Masciulli A, Bacigalupo A, Bregante S, Pavoni C, Finazzi MC, et al. Busulfan- or Thiotepa-Based Conditioning in Myelofibrosis: A Phase II Multicenter Randomized Study From the GITMO Group. Biol Blood Marrow Transplant (2019) 25(5):932-40. doi: 10.1016/j.bbmt.2018. 12.064

37. Bartelink IH, Lalmohamed A, van Reij EM, Dvorak CC, Savic RM, Zwaveling J, et al. Association of Busulfan Exposure With Survival and Toxicity After Haemopoietic Cell Transplantation in Children and Young Adults: A Multicentre, Retrospective Cohort Analysis. Lancet Haematol (2016) 3(11): e526-e36. doi: 10.1016/s2352-3026(16)30114-4

38. Tefferi A, Vaidya R, Caramazza D, Finke C, Lasho T, Pardanani A. Circulating Interleukin (IL)-8, IL-2r, IL-12, and IL-15 Levels Are Independently Prognostic in Primary Myelofibrosis: A Comprehensive Cytokine Profiling Study. J Clin Oncol (2011) 29(10):1356-63. doi: 10.1200/jco.2010.32.9490

39. Burnham AJ, Daley-Bauer LP, Horwitz EM. Mesenchymal Stromal Cells in Hematopoietic Cell Transplantation. Blood Adv (2020) 4(22):5877-87. doi: 10.1182/bloodadvances.2020002646

40. Le Blanc K, Frassoni F, Ball L, Locatelli F, Roelofs H, Lewis I, et al. Mesenchymal Stem Cells for Treatment of Steroid-Resistant, Severe, Acute Graft-Versus-Host Disease: A Phase II Study. Lancet (2008) 371(9624):157986. doi: 10.1016/s0140-6736(08)60690-x

41. Kallekleiv M, Larun L, Bruserud $\varnothing$, Hatfield KJ. Co-Transplantation of Multipotent Mesenchymal Stromal Cells in Allogeneic Hematopoietic Stem Cell Transplantation: A Systematic Review and Meta-Analysis. Cytotherapy (2016) 18(2):172-85. doi: 10.1016/j.jcyt.2015.11.010

42. Lee SH, Lee MW, Yoo KH, Kim DS, Son MH, Sung KW, et al. CoTransplantation of Third-Party Umbilical Cord Blood-Derived MSCs Promotes Engraftment in Children Undergoing Unrelated Umbilical Cord Blood Transplantation. Bone Marrow Transplant (2013) 48(8):1040-5. doi: $10.1038 / \mathrm{bmt} .2013 .7$ 
43. Wang X, Zhang M, He P. Pre-Infusion Single-Dose Mesenchymal Stem Cells Promote Platelet Engraftment and Decrease Severe Acute Graft Versus Host Disease Without Relapse in Haploidentical Peripheral Blood Stem Cell Transplantation. J Int Med Res (2020) 48(5):300060520920438. doi: 10.1177/0300060520920438

44. Liu Z, Zhang Y, Xiao H, Yao Z, Zhang H, Liu Q, et al. Cotransplantation of Bone Marrow-Derived Mesenchymal Stem Cells in Haploidentical Hematopoietic Stem Cell Transplantation in Patients With Severe Aplastic Anemia: An Interim Summary for a Multicenter Phase II Trial Results. Bone Marrow Transplant (2017) 52(5):704-10. doi: 10.1038/bmt.2016.347

45. Ning H, Yang F, Jiang M, Hu L, Feng K, Zhang J, et al. The Correlation Between Cotransplantation of Mesenchymal Stem Cells and Higher Recurrence Rate in Hematologic Malignancy Patients: Outcome of a Pilot Clinical Study. Leukemia (2008) 22(3):593-9. doi: 10.1038/sj.leu.2405090

46. Zhao K, Lou R, Huang F, Peng Y, Jiang Z, Huang K, et al. Immunomodulation Effects of Mesenchymal Stromal Cells on Acute Graft-Versus-Host Disease After Hematopoietic Stem Cell Transplantation. Biol Blood Marrow Transplant (2015) 21(1):97-104. doi: 10.1016/j.bbmt.2014.09.030

47. Siragusa S, Passamonti F, Cervantes F, Tefferi A. Survival in Young Patients With Intermediate- / High-Risk Myelofibrosis: Estimates Derived From Databases for Non Transplant Patients. Am J Hematol (2009) 84(3):140-3. doi: 10.1002/ajh.21342

48. Park SK, Won JH, Kim HJ, Bae SB, Kim CK, Lee KT, et al. Co-Transplantation of Human Mesenchymal Stem Cells Promotes Human CD34+ Cells
Engraftment in a Dose-Dependent Fashion in NOD/SCID Mice. J Korean Med Sci (2007) 22(3):412-9. doi: 10.3346/jkms.2007.22.3.412

49. Tobin LM, Healy ME, English K, Mahon BP. Human Mesenchymal Stem Cells Suppress Donor CD4(+) T Cell Proliferation and Reduce Pathology in a Humanized Mouse Model of Acute Graft-Versus-Host Disease. Clin Exp Immunol (2013) 172(2):333-48. doi: 10.1111/cei.12056

Conflict of Interest: The authors declare that the research was conducted in the absence of any commercial or financial relationships that could be construed as a potential conflict of interest.

Publisher's Note: All claims expressed in this article are solely those of the authors and do not necessarily represent those of their affiliated organizations, or those of the publisher, the editors and the reviewers. Any product that may be evaluated in this article, or claim that may be made by its manufacturer, is not guaranteed or endorsed by the publisher.

Copyright (C) 2022 Wang, Xu, Wang, Zhang, Liu, Zhou, Wang, Zhang, Tang, Fu, Miao and $W u$. This is an open-access article distributed under the terms of the Creative Commons Attribution License (CC BY). The use, distribution or reproduction in other forums is permitted, provided the original author(s) and the copyright owner(s) are credited and that the original publication in this journal is cited, in accordance with accepted academic practice. No use, distribution or reproduction is permitted which does not comply with these terms. 